\title{
On Recent Existence Theorems in the Theory of Optimization ${ }^{1}$
}

\author{
L. CESARI ${ }^{2}$ AND M. B. SURYANARAYANA ${ }^{3}$
}

\begin{abstract}
A condition recently proposed is shown to imply the weak compactness in $H^{1,1}$ and actually is equivalent to another condition previously proposed by the authors. Once compactness is proved, then existence theorems follow from lower closure theorems also previously proved by the authors, and extended to Pareto problems. The present analysis adds to the recent work of Goodman concerning the equivalence of seminormality conditions with concepts of convex analysis and lattice theory.
\end{abstract}

Key Words. Lower closure, weak compactness, existence theorems, convex duality, property $(Q)$, Lipschitz type conditions.

\section{Introduction}

In a recent paper by Cecconi, a weak compactness theorem in $H^{1,1}$ is reported as having been proved by Rockafellar on the basis of convex analysis and functional analysis, and based on a condition that we call here (G3). Actually, this condition is a variant of the Nagumo type and analogous growth conditions, and indeed we first prove (Section 2) that condition (G3) is equivalent to a condition of that type, say $(\mathrm{G} 2)$, that we have used for the same scope in previous papers.

Once the weak compactness theorem is proved, the ensuing existence theorems in the theory of optimization are well-known consequences of lower-closure theorems which we proved in the past years (e.g., Refs. 1, 2), where (among other points) no condition $(Q)$ is explicitly needed (see Section 3). Actually we further extended these lower-closure theorems and ensuing existence theorems to functionals taking values in any $R^{n}$, or any reflexive Banach space, where a partial ordering has been defined by a given closed convex cone (Refs. 3-6).

\footnotetext{
${ }^{1}$ Paper received February 2, 1979.

${ }^{2}$ Professor, Department of Mathematics, University of Michigan, Ann Arbor, Michigan.

${ }^{3}$ Professor, Department of Mathematics, Eastern Michigan University, Ypsilanti, Michigan.
} 
Actually, as a consequence of the weak convergence of the derivatives property, and a well-known remark by De la Valle Poussin, a Nagumo-type growth condition holds, under which suitable auxiliary sets possess property $(Q)$, and this condition-whether we explicitly name it or not-enters in the proof of the lower-closure theorems. We point out here that property $(Q)$ has been equivalently expressed by Cesari (Refs. 7,8 ) in terms of Tonelli and McShane seminormality conditions and by Goodman (Ref. 9) in terms of duality operations in convex analysis and lattice theory in point-set topology (see Section 5 for Goodman's equivalence statements).

In recent work of Berkovitz and Bates (see Remark 6.1), certain Lipschitz-type conditions are used in place of property $(Q)$, claiming that these conditions are independent of property $(Q)$. In Remark 6.1, we point out that the contrary is true, since their Lipschitz condition is a particular case of an analytic condition, namely, property $(D)$ (see Section 6) studied long ago by Cesari and Suryanarayana (Ref. 10), which implies a suitable form of property $(Q)$. It is relevant that this property $(D)$ is valid even in Hilbert spaces and Banach spaces (Ref. 3). Finally, in Remark 3.2, we correct an erroneous statement by Olech in one of his recent reviews.

\section{Weak Compactness}

Let us consider any family

$$
\mathfrak{S}=\{\eta(t), x(t), a \leqslant t \leqslant b\}
$$

of pairs of functions, $\eta(t)$ scalar and $L$-integrable, and $x(t)=\left(x_{1}, \ldots, x_{n}\right)$ absolutely continuous in intervals $[a, b]$, not necessarily the same, but within a fixed finite interval or $-\infty<a_{0} \leqslant a<b \leqslant b_{0}<+\infty$. There are wellknown growth conditions which guarantee compactness properties of the class $\{x\}$ of all elements $x(t), a \leqslant t \leqslant b$, appearing in $(\mathbb{E}$. We name here a few.

(G1) There are a constant $M \geqslant 0$ and a scalar function $\Phi(\xi), 0 \leqslant \xi<$ $+\infty$, bounded below and with $\Phi(\xi) / \xi \rightarrow \infty$ as $\xi \rightarrow \infty$, such that

$$
\text { (a) } \int_{a}^{b} \eta(t) d t \leqslant M
$$

and

(b) $\eta(t) \geqslant \Phi\left(\left|x^{\prime}(t)\right|\right), \quad a \leqslant t \leqslant b$,

for all pairs $\eta, x$ of class 5 . 
(G2) There are a constant $M \geqslant 0$ and, for every $\epsilon>0$, a scalar function $\Psi_{\epsilon}(t) \geqslant 0, L$-integrable in $\left[a_{0}, b_{0}\right]$, such that

(a) $\int_{a}^{b} \eta(t) d t \leqslant M$, and

(b) $\left|x^{\prime}(t)\right| \leqslant \Psi_{\epsilon}(t)+\epsilon \eta(t), \quad a \leqslant t \leqslant b$,

for all pairs $\eta, x$ in the class $(5$.

(G3) There are a constant $M \geqslant 0$ and, for every $p=\left(p_{1}, \ldots, p_{n}\right) \in R^{n}$, a scalar function $\phi_{p}(t) \geqslant 0, L$-integrable in $\left[a_{0}, b_{0}\right]$, such that

(a) $\int_{a}^{b} \eta(t) d t \leqslant M$, and

(b) $\eta(t) \geqslant\left(p, x^{\prime}(t)\right)-\phi_{p}(t), \quad a \leqslant t \leqslant b$,

for all pairs $\eta, x$ of the class $C$.

Theorem 2.1. Under either condition (G1), (G2), or (G3), the class $\{x\}$ is equiabsolutely continuous and the class $\left\{x^{\prime}\right\}$ is equiabsolutely integrable. Thus if, in addition, the class $\{x\}$ is equibounded, then the same class $\{x\}$ is relatively weakly compact in $H^{1,1}$.

This is essentially the Tonelli-Nagumo statement under condition (G1) (see, for example, Ref. 11). The same statement was essentially proved by Cesari, La Palm, and Nishiura (Ref. 12) under condition (G2), and for the sake of simplicity we give a direct proof of this in the Appendix below. (See also Ref. 15.) Condition (G3) is the one mentioned by Cecconi (Ref. 13, Theorem 6, p. 296) as reported by Rockafellar (Ref. 14). Here, under (i) we give a simple direct proof of Theorem 2.1 under condition (G3). Under (ii), we prove that (G3) is equivalent to (G2). Condition (G1) implies (G2), but $(\mathrm{G} 2)$ is actually more general than (G1). For instance, for $\eta(t)=t^{\alpha} x^{\prime 2}$, $0 \leqslant t \leqslant 1,0<\alpha<1$, condition (G2) is satisfied, but (G1) is not. Condition (G2) has been extensively used in Refs. 3, 12, 16, and elsewhere. Thus, condition (G2) applied to the integral

$$
I=\int_{0}^{1} t^{\alpha} x^{\prime 2} d t, \quad x(0)=1, \quad x(1)=0, \quad t x^{\prime 2},
$$

allows one to separate the case $\alpha \geqslant 1$ for which $I$ has no absolute minimum from the case $0<\alpha<1$ for which $I$ has an absolute minimum. Condition (G3) also was used in Ref. 16 and other papers.

Proof. (i). Direct Proof of Theorem 2.1 under Condition (G3). Let $\varphi(t), \psi(t), a_{0} \leqslant t \leqslant b_{0}$, be the nonnegative $L$-integrable functions in (G3)(b) 
corresponding to the two unit vectors

$$
p=u_{1}=(1,0, \ldots, 0) \quad \text { and } \quad p=-u_{1}=(-1,0, \ldots, 0) .
$$

Then, for any pair $\eta, x$ in $\mathbb{E}$, we have

$$
x_{1}^{\prime}(t) \leqslant \eta(t)+\varphi(t), \quad-x_{1}^{\prime}(t) \leqslant \eta(t)+\psi(t), \quad a \leqslant t \leqslant b,
$$

and hence

$$
\left|x_{1}^{\prime}(t)\right| \leqslant \eta(t)+\varphi(t)+\psi(t), \quad a \leqslant t \leqslant b .
$$

Thus,

$$
\eta(t)+\varphi(t)+\psi(t) \geqslant 0, \quad a \leqslant t \leqslant b .
$$

Let

$$
M_{1}=\int_{a}^{b}(\varphi(t)+\psi(t)) d t
$$

Now, given $\epsilon>0$, let $N$ be an integer, such that

$$
N^{-1} n M \leqslant \epsilon / 3, \quad N^{-1} n M_{1} \leqslant \epsilon / 3 .
$$

If $u_{i}, v_{i}$ denote the unit vectors

$$
u_{i}=\left(\delta_{i j}, j=1,2, \ldots, n\right), \quad v_{i}=\left(-\delta_{i j}, j=1, \ldots, n\right),
$$

then, for $p=N u_{i}$ and $p=N v_{i}$, there are functions

$$
\Phi_{i}(t) \geqslant 0, \quad \Psi_{i}(t) \geqslant 0,
$$

$L$-integrable in $\left[a_{0}, b_{0}\right]$, such that

$N x_{i}^{\prime}(t) \leqslant \eta(t)+\Phi_{i}(t), \quad-N x_{i}^{\prime}(t) \leqslant \eta(t)+\Psi_{i}(t), \quad a \leqslant t \leqslant b, \quad i=1, \ldots, n$, and hence

$$
N\left|x_{i}^{\prime}(t)\right| \leqslant \eta(t)+\Phi_{i}(t)+\Psi_{i}(t), \quad a \leqslant t \leqslant b, \quad i=1, \ldots, n .
$$

Then,

$$
\Phi(t)=\sum_{i=1}^{n} \Phi_{i}(t), \quad \Psi(t)=\sum_{i=1}^{n} \Psi_{i}(t)
$$

are $L$-integrable in $\left[a_{0}, b_{0}\right]$, and we also have

$$
N\left|x^{\prime}(t)\right| \leqslant n \eta(t)+\Phi(t)+\Psi(t), \quad a \leqslant t \leqslant b .
$$


If $E$ denotes any measurable subset of $[a, b]$, we have, from inequalities (1)-(2),

$$
\begin{aligned}
\int_{E}\left|x^{\prime}(t)\right| d t \leqslant & N^{-1} n \int_{E} \eta(t) d t+N^{-1} n \int_{E}(\Phi(t)+\Psi(t)) d t \\
\leqslant & N^{-1} n \int_{E}[\eta(t)+\varphi(t)+\psi(t)] d t+N^{-1} n \int_{E}(\Phi(t)+\Psi(t)) d t \\
\leqslant & N^{-1} n \int_{a}^{b} \eta(t) d t+N^{-1} n \int_{a}^{b}(\varphi(t)+\psi(t)) d t \\
& +N^{-1} n \int_{E}(\Phi(t)+\Psi(t)) d t .
\end{aligned}
$$

Since $\Phi+\Psi$ is $L$-integrable, there is $\delta>0$, such that meas $E \leqslant \delta$ implies that

$$
\int_{E}(\Phi+\Psi) d t \leqslant \epsilon / 3
$$

and hence

$$
\int_{E}\left|x^{\prime}(t)\right| d t \leqslant \epsilon / 3+\epsilon / 3+\epsilon / 3=\epsilon .
$$

We have proved the equiabsolute integrability of the class $\left\{x^{\prime}\right\}$. From here, the equiabsolute continuity of the class $\{x\}$ follows immediately. If we know that the class $\{x\}$ is equibounded, then the compactness of $\{x\}$ in $C$ follows from the Ascoli theorem, and the weak compactness of $\{x\}$ in $H^{1,1}$ follows from the Dunford-Pettis theorem.

Proof. (ii). Proof that (G3) implies (G2). If (G3) is satisfied, then, given $\epsilon>0$, let us consider the $2 n$ points $p \in R^{n}$ defined by $p= \pm \epsilon^{-1} n\left(\delta_{i s}\right.$, $s=1, \ldots, n), i=1, \ldots, n$. Let $\phi_{p}(t) \geqslant 0$ be the corresponding $2 n$ locally integrable functions, let $\phi(t)$ denote their sum, and take

$$
\Psi_{\varepsilon}(t)=\phi(t)
$$

Then

$$
\eta(t) \geqslant \pm \epsilon^{-1} n x_{i}^{\prime}(t)-\phi_{p}(t)
$$

for each of the $2 n$ points above, and hence

$$
\left|x^{\prime}(t)\right| \leqslant \sum_{i}\left|x_{i}^{\prime}(t)\right| \leqslant \epsilon \eta(t)+\Psi_{\epsilon}(t)
$$

for all $t$. Thus, (G3) implies (G2). 
Proof that (G2) implies (G3). Given $p \in R^{n}$ take $\epsilon>0$ such that $1 / \epsilon \geqslant|p|$. By $(\mathrm{G} 2)$ there is $\Psi_{\epsilon}(t) \geqslant 0, \Psi_{\epsilon}$ locally integrable, such that $\left|x^{\prime}(t)\right| \leqslant \Psi_{\epsilon}(t)+\epsilon \eta(t)$, that is,

$$
\eta(t) \geqslant(1 / \epsilon)\left|x^{\prime}(t)\right|-(1 / \epsilon) \Psi_{\epsilon}(t) \geqslant\left(p, x^{\prime}(t)\right)-\phi_{p}(t),
$$

where $\phi_{p}(t)=(1 / \epsilon) \Psi_{\epsilon}(t)$. Thus (G2) implies (G3).

A direct proof of Theorem 2.1 under condition (G2) is given in the Appendix.

Remark 2.1. The arguments underlying Theorem 2.1 apply as well to any family

$$
\mathcal{E}=\{\eta(t), \xi(t), t \in G\}
$$

of pairs of functions

$$
\eta \in L_{1}(G), \xi \in\left(L_{1}(G)\right)^{n}, \quad \text { with } \int_{G} \eta(t) d t \leqslant M,
$$

where $G$ is any measurable bounded subset of $R^{\nu}$. Condition (G1) then becomes

$$
\eta(t) \geqslant \phi(|\xi(t)|), \quad t \in G
$$

for all pairs $(\eta, \xi)$ in $\subseteq$. Condition (G2) then becomes: given $\epsilon>0$, there is some $\psi_{\epsilon}(t) \geqslant 0, t \in G, \psi_{\epsilon} \in L_{1}(G)$, such that

$$
|\xi(t)| \leqslant \Psi_{\epsilon}(t)+\epsilon \eta(t), \quad t \in G,
$$

for all pairs $(\eta, \xi)$ in $\left(5\right.$. Condition (G3) then becomes: given $p \in R^{n}$, there is $\phi_{p}(t) \geqslant 0, t \in G, \phi_{p} \in L_{1}(G)$, such that

$$
\eta(t) \geqslant(p, \xi(t))-\phi_{p}(t), \quad t \in G
$$

for all pairs $(\eta, \xi)$ in $\mathfrak{E}$. Under either hypothesis $(\mathrm{G} 1),(\mathrm{G} 2)$, or (G3), the class $\{\xi(t)\}$ is equiabsolutely integrable in $G$ and hence relatively weakly compact in $L_{1}(G)$.

\section{Some Lower-Closure and Existence Theorems}

We briefly state here a simple version of a lower-closure theorem. For $t \in[a, b]$, let $A(t)$ denote a closed subset of the $y$-space $R^{n}$; let

$$
A=[(t, y) \mid a \leqslant t \leqslant b, y \in A(t)] \subset R^{1+n},
$$

for every $(t, y) \in A$ let $Q(t, y)$ denote a nonempty closed subset of the $z$-space $R^{n}$; and let

$$
M=[(t, y, z) \mid(t, y) \in A, z \in Q(t, y)] \subset R^{1+2 n} .
$$


Let $F_{0}(t, y, z)$ denote a real-valued function defined on $M, F_{0}<+\infty$ on $M$, and let $F_{0}(t, y, z)$ denote also the extended function defined by taking $F_{0}=+\infty$ in $R^{1+2 n}-M$. Let us assume that the extended function $F_{0}$ is measurable in $t$ for all $(y, z)$ and that, for almost all $\vec{t}, F_{0}(\vec{t}, y, z)$ is lower semicontinuous in $(y, z)$ and convex in $z$.

Theorem 3.1. Lower Closure Theorem. If $\xi(t), y(t), \eta_{k}(t), \xi_{k}(t), y_{k}(t)$, $\lambda(t), \lambda_{k}(t), t \in G=[a, b], k=1,2, \ldots$, are measurable functions, $\xi, \xi_{k} \in$ $\left(L_{1}(G)\right)^{r}, \eta_{k} \in L_{1}(G)$, such that $y_{k} \rightarrow y$ in measure in $G, \xi_{k} \rightarrow \xi$ weakly in $\left(L_{1}(G)\right)^{r}$ as $k \rightarrow \infty$, with

$$
\begin{gathered}
y_{k}(t) \in A(t), \quad \begin{array}{c}
\xi_{k}(t) \in Q\left(t, y_{k}(t)\right), \quad \begin{array}{c}
\eta_{k}(t) \geqslant F_{0}\left(t, y_{k}(t), \xi_{k}(t)\right), \\
t \in G \text { a.e., } \quad k=1,2, \ldots,
\end{array} \\
-\infty \leqslant i=\lim _{k \rightarrow \infty} \inf \int_{G} \eta_{k}(t) d t<+\infty,
\end{array} \\
\eta_{k}(t) \geqslant \lambda_{k}(t), \quad \lambda, \lambda_{k} \in L_{1}(G), \quad \lambda_{k} \rightarrow \lambda \text { weakly in } L_{1}(G),
\end{gathered}
$$

then there is a function $\eta(t), t \in G, \eta \in L_{1}(G)$, such that

$$
\begin{aligned}
y(t) \in A(t), \quad \xi(t) \in Q(t, y(t)), \quad \eta(t) & \geqslant F_{0}(t, y,(t), \xi(t)), \\
t & \in G \text { a.e., } \quad \int_{G} \eta(t) d t \leqslant i .
\end{aligned}
$$

For this theorem, see Cesari (Ref. 1) and Cesari and Suryanarayana (Ref. 2). For extensions to multidimensional $t$ or $t, y, f_{0}$ valued in Banach spaces, see Refs. 2, 3, 5.

For every $(t, y) \in A$, let $\tilde{Q}(t, y)$ denote the set of all $\left(z^{0}, z\right) \in R^{n+1}$, with

$$
z^{0} \geqslant F_{0}(t, y, z), \quad z \in Q(t, y) .
$$

The condition concerning $F_{0}$ above can be equivalently expressed by requiring that $F_{0}$ be measurable in $t$ for all $(y, z)$, that $A$ be closed, and that, for almost all $\bar{t}$, the sets $\tilde{Q}(\bar{t}, y)$ have property $(K)$ with respect to $y$ in $A(\bar{t})$ and are convex (see Section 4 below).

For lower-closure theorems for Banach valued functions $y(t)$ see Cesari (Refs. 3, 5). Of course, Theorem 3.1 holds even for functions defined on any bounded measurable subset $G$ of $R^{\nu}$ or any finite measure space.

Remark 3.1. Note that, in the lower-closure Theorem 3.1, if we know that the functions $\lambda_{k}(t), t \in G$, can be taken in the form

$$
\lambda_{k}(t)=\left(p, \xi_{k}(t)\right)-\phi_{p}(t), \quad t \in G, \quad k=1,2, \ldots,
$$


for every fixed $p \in R^{n}$ and corresponding

$$
\phi_{p}(t) \geqslant 0, \quad t \in G, \phi_{p} \in L_{1}(G),
$$

then, by Remark 2.1 , the sequence $\lambda_{k}$ certainly has a subsequence which is weakly convergent in $\left(L_{1}(G)\right)^{n}$ as required.

Remark 3.2. Note that the lower-closure Theorem 3.1 has been proved in Ref. 1 for functions $\xi(t), \xi_{k}(t)$ having their values in a finitedimensional space $\boldsymbol{R}^{r}$, as stated. For functions $\xi, \xi_{k}$ having their values in a real Banach space $B$, we have proved in Ref. 2, Refs. 3, Part I, and Refs. 4, 16,17 an analogous statement, namely, the same as Theorem 3.1 with the following modified requirements:

$$
\begin{array}{r}
\xi(t), y(t), \eta_{k}(t), \xi_{k}(t), y_{k}(t), \mu(t), \mu_{k}(t), p_{k}(t), \lambda(t), \lambda_{k}(t), t \in G, \\
k=1,2, \ldots,
\end{array}
$$

are measurable functions, $\xi, \xi_{k}, p_{k} \in L_{1}(G, B), \eta_{k}, \mu_{k}, \mu, \lambda_{k}, \lambda \in L_{1}(G, R)$, $y(t), y_{k}(t) \in A(t)$, such that

$$
\begin{gathered}
\eta_{k}(t) \geqslant F_{0}\left(t, y_{k}(t), \xi_{k}(t)\right), \quad \mu_{k}(t) \geqslant F_{0}\left(t, y_{k}(t), p_{k}(t)\right), \\
\xi_{k}(t) \in Q\left(t, y_{k}(t)\right), \quad p_{k}(t) \in Q\left(t, y_{k}(t)\right), \quad t \in G \text { a.e., } \quad k=1,2, \ldots, \\
-\infty<i=\lim _{k \rightarrow \infty} \inf \int_{G} \eta_{k}(t) d t<+\infty, \quad \eta_{k}(t) \geqslant \lambda_{k}(t), \\
t \in G, \quad k=1,2, \ldots,
\end{gathered}
$$

$\xi_{k}(t) \rightarrow \xi(t)$ weakly in $L_{1}(G, B), \quad y_{k}(t) \rightarrow y(t)$ in measure in $G$,

$$
p_{k}(t) \rightarrow p(t) \text { strongly in } L_{1}(G, B), \quad \mu_{k}(t) \rightarrow \mu(t) \text { weakly in } L_{1}(G, R) \text {, }
$$

$$
\lambda_{k}(t) \rightarrow \lambda(t) \text { weakly in } L_{1}(G, R) .
$$

Then, there is a function $\eta(t), t \in G, \eta \in L_{1}(G, R)$, such that

$$
\eta(t) \geqslant F_{0}(t, x(t), \xi(t)), \quad t \in G \text { a.e., } \quad \int_{G} \eta(t) d t \leqslant i \text {. }
$$

As we proved in the quoted papers, we may allow $G$ to be any metric space and also a finite complete measure space $(G, \alpha, \mu)$, and thus $d \mu$ may replace $d t$ above, and $y, y_{k}$ may take their values in any metric space $(Y, d)$.

Remark 3.3. Olech (Ref. 19), in his review of Ref. 2, has stated that the existence of sequences $\mu_{k}, p_{k}$ (with $\mu_{k} \rightarrow \mu$ weakly in $L_{1}$, and $p_{k} \rightarrow p$ strongly in $L_{1}$ ) is a consequence of the other hypotheses in Theorem 4.1, p. 172, Ref. 2, that is, the proposition in Remark 2.1 above. Olech's statement is not true, as Example 5.4,p. 178 of the same paper (Ref. 2), exactly shows. 
However, in finite-dimensional spaces, as shown in Ref. 4, it is possible to prove the lower-closure theorem without this specific assumption regarding the existence of a sequence $\mu_{k}(t), p_{k}(t)$, with

$$
p_{k}(t) \in Q\left(t, y_{k}(t)\right), \quad \mu_{k}(t) \geqslant F_{0}\left(t, y_{k}(t), p_{k}(t)\right),
$$

such that $p_{k}$ is strongly convergent and $\mu_{k}$ is weakly convergent. Such sequences may not exist even if the upper semicontinuity property $(K)$ in $y$ of the sets $Q(t, y)$ is assumed. If such sequences do exist, then the original minimizing sequences $\eta_{k}, \xi_{k}$ can be modified suitably so as to lie in equibounded sets $Q^{*}\left(t, y_{k}(t)\right)$, which would then have property $(Q)$.

Remark 3.4. As in Remark 2.1, we mention here another version of the lower-closure Theorem 3.1, which we shall use in Section 5 below, and for which we refer to Ref. 10 and Ref. 3, Part I, for proofs. Namely, the same Theorem 3.1 holds with the following modified requirements:

$\xi(t), y(t), \eta_{k}(t), \xi_{k}(t), y_{k}(t), \bar{\eta}_{k}(t), \bar{\xi}_{k}(t), \lambda(t), \lambda_{k}(t), \quad t \in G, \quad k=1,2, \ldots$, are measurable functions, $\xi, \xi_{k}, \bar{\xi}_{k} \in L_{1}(G, B), \eta_{k}, \bar{\eta}_{k}, \lambda_{k}, \lambda \in L_{1}(G, R)$, $y(t), y_{k}(t) \in A(t)$, such that

$$
\begin{gathered}
\eta_{k}(t) \geqslant F_{0}\left(t, y_{k}(t), \xi_{k}(t)\right), \quad \bar{\eta}_{k}(t) \geqslant F_{0}\left(t, y(t), \bar{\xi}_{k}(t)\right), \\
\xi_{k}(t) \in Q\left(t, y_{k}(t)\right), \quad \bar{\xi}_{k}(t) \in Q(t, y(t)), \quad \eta_{k}(t), \bar{\eta}_{k}(t) \geqslant \lambda_{k}(t), \quad t \in G, \\
-\infty<i=\lim _{k \rightarrow \infty} \text { inf } \int_{G} \eta_{k}(t) d t<+\infty, \\
\xi_{k}(t) \rightarrow \xi(t) \text { weakly in } L_{1}(G, B), \quad y_{k}(t) \rightarrow y(t) \text { in measure in } G, \\
\lambda_{k}(t) \rightarrow \lambda(t) \text { weakly in } L_{1}(G, R), \\
\delta_{k}(t)=\xi_{k}(t)-\bar{\xi}_{k}(t) \rightarrow 0 \text { strongly in } L_{1}(G, B), \\
\delta_{k}^{0}(t)=\eta_{k}(t)-\bar{\eta}_{k}(t) \rightarrow 0 \text { strongly in } L_{1}(G, R) .
\end{gathered}
$$

Then, there is a function $\eta(t), t \in G, \eta \in L_{1}(G, R)$, such that

$$
\eta(t) \geqslant F_{0}(t, y(t), \xi(t)), \eta(t) \in Q(t, y(t)), \quad t \in G \text { a.e., } \quad \int_{G} \eta(t) d t \leqslant i
$$

We can now state an existence theorem, in which again for the sake of simplicity we assume $t$ scalar, $y \in R^{n}, A$ compact, and $F_{0}$ also scalar. Thus, we are concerned here with a functional

$$
I[y]=g\left(t_{1}, y\left(t_{1}\right), t_{2}, y\left(t_{2}\right)\right)+\int_{t_{1}}^{t_{2}} F_{0}\left(t, y(t), y^{\prime}(t)\right) d t,
$$

where $g\left(t_{1}, y_{1}, t_{2}, y_{2}\right)$ is a scalar function defined on a subset $B$ of $R^{2+2 n}$, 
$F_{0}(t, y, z)$ is a scalar function defined on a set

$$
M=[(t, y, z) \mid(t, y) \in A, z \in Q(t, y)]
$$

of the tyz-space and extended to $R^{1+2 n}$ as stated above. We may require on $F_{0}$ one of the following growth conditions:

(g1) there is a scalar function $\phi(\xi), 0 \leqslant \xi<+\infty$, bounded below with $\phi(\xi) / \xi \rightarrow \infty$, such that $F_{0}(t, y, z) \geqslant \phi(|z|)$ for all $(t, y, z)$;

(g2) for every $\epsilon>0$, there is a scalar function $\psi_{\epsilon}(t) \geqslant 0$, locally integrable, such that $|z| \leq \psi_{\epsilon}(t)+\epsilon F_{0}(t, y, z)$;

(g3) for every $p \in R^{n}$, there is a scalar function $\phi_{p}(t)$, locally integrable, such that $F_{0}(t, y, z) \geqslant(p, z)-\phi_{p}(t)$ for all $(t, y, z)$.

Let $\Omega$ be a nonempty closed class of absolutely continuous functions $y(t)=\left(y_{1}, \ldots, y_{n}\right), \quad t_{1} \leqslant t \leqslant t_{2}$, with $(t, y(t)) \in A, \quad\left(t_{1}, y\left(t_{1}\right), t_{2}, y\left(t_{2}\right)\right) \in B$; $F_{0}\left(\cdot, y(\cdot), y^{\prime}(\cdot)\right)$ is $L$-integrable in $\left[t_{1}, t_{2}\right]$. The property of closedness needed here is a very mild one, that is, every limit element $y$ in the weak topology of $H^{1,1}$ which has the properties just stated belongs to $\Omega$. Thus, the class $\Omega$ of all $y$ with the properties stated is closed. The existence theorem below certainly holds in the class of all $y$ with the properties stated.

Theorem 3.2. Existence Theorem for Problems of Calculus of Variations and Optimal Control Theory. Let $A$ be compact, $B$ closed, and let us assume that $g$ is lower semicontinuous on $B$, that the extended function $F_{0}(t, y, z)$ is measurable in $t$ for all $(y, z)$, and that, for almost all $t, F_{0}(t, y, z)$ is lower semicontinuous in $(y, z)$ and convex in $z$. Let us assume that one of the growth hypotheses (G1), (G2), or (G3) holds. Then, the functional (3) has an absolute minimum in $\Omega$.

If no condition $(G)$ holds, the conclusion is still valid provided we know that the class $\Omega$ is weakly compact in $H^{1,1}$ and $F_{0}$ satisfies one of the following conditions:

(L1) $F_{0}(t, y, z) \geqslant-\psi(t)$, for some locally integrable function $\psi \geqslant 0$;

(L2) $F_{0}(t, y, z) \geqslant-\psi(t)-c|z|$, for some constant $c$ and $\psi$ as in (L1);

(L3) $F_{0}(t, y, z) \geqslant-\psi(t)-(\varphi(t), z)$, for $\psi$ as in L1 and some bounded measurable $\varphi$;

(L4) $F_{0}(t, y, z)$ is defined at least in $A \times B_{0}$, where $B_{0}$ is the closed unit ball in $R^{n}$ and $F_{0}(t, y, z) \geqslant \beta$ in $A \times B_{0}$ and $F_{0}(t, y, 0) \leqslant \alpha$ in $A$ for some constants $\alpha \leqslant \beta$.

The condition (L4) is certainly satisfied if $F_{0}$ is continuous on $A \times B_{0}$.

The extension of Theorem 3.2 to the case of $A$ being not compact, but closed, is only technical. For extensions to the case where $t$ is multidimensional or $t, y, F_{0}$ take their values in Banach spaces, we refer to Cesari (Ref. 3, Part I) and Cesari and Suryanarayana (Refs. 2, 4). 


\section{Some Upper Semicontinuity Properties of Set-Valued Maps}

Given any set $Z$ in a linear topological space $Y$, we denote by $\mathrm{cl} Z$, bd $Z$, co $Z$ the closure of $Z$, the boundary of $Z$, and the convex hull of $Z$, respectively. Thus, cl co $Z$ denotes the closure of the convex hull of $Z$.

Let $x \rightarrow Q(x), x \in X, Q(x) \subset Y$ be a set-valued map from a metric space $X$ to a linear topological space $Y$. Let $x_{0}$ be a point of $X$.

Kuratowski's concept of upper semicontinuity is relevant. We say that the map $x \rightarrow Q(x)$ has property $(K)$ at $x_{0}$ (Kuratowski), provided

$$
Q\left(x_{0}\right)=\bigcap_{\delta>0} \mathrm{~d} \cup\left[Q(x), x \in N_{\delta}\left(x_{0}\right)\right] .
$$

Here, $N_{\delta}\left(x_{0}\right)$ denotes the $\delta$-neighborhood of $x_{0}$ in $X$. Note that $Q\left(x_{0}\right)$, as the intersection of closed sets, is certainly closed.

We shall need also the following variant. We say that the map $x \rightarrow Q(x)$ has property $(Q)$ at $x_{0}$, provided

$$
Q\left(x_{0}\right)=\bigcap_{\delta>0} \operatorname{clco} \cup\left[Q(x), x \in N_{\delta}\left(x_{0}\right)\right]
$$

(Cesari, Ref. 20). Here, $Q\left(x_{0}\right)$, as the intersection of closed convex sets, is certainly closed and convex.

We say that the map has property $(K)$ or $(Q)$ in $X$ if it has such property at every point $x_{0}$ of $X$. For brevity, we may also say that the sets $Q(x)$ have property $(K)$ or $(Q)$. The indication "with respect to," will be needed if the sets depend also on other parameters which then are kept constant. It is well known that property $(Q)$ implies property $(K)$. Moreover, the map $x \rightarrow Q(x)$ has the property $(K)$ in $X$ if and only if its graph

$$
[(x, y) \mid x \in X, y \in Q(x)]
$$

is closed in the product space $X \times Y$.

We are concerned here with the situation in which a closed set $A$ is given, say in $R^{\nu}$, for every $x \in A$ a subset $Q(x)$ of $R^{n}$ is given, $M \subset R^{\nu+n}$ is the set of all $(x, u)$ with $x \in A, z \in Q(x)$, and $T(x, u)$ is a real-valued function on $M$. For every $x \in A$, let $\tilde{Q}(x)$ denote the subsets of $R^{n+1}$ defined by

$$
\tilde{Q}(x)=\left[\left(z^{0}, u\right): z^{0} \geqslant T(x, u), u \in Q(x)\right] .
$$

We may extend the function $T$ outside $M$ by taking $T=+\infty$ in $R^{\nu+n}-M$. Cesari has proved that the sets $\dot{Q}(x)$ have property $(Q)$ (with respect to $x$ ) in $A$ if and only if $T(x, u)$ has everywhere in $M$ the Tonelli-McShane seminormality property expressed as usual in terms of supporting hyperplanes to $\tilde{Q}(x)$ in $R^{n+1}$ [Cesari, Refs. 7, 15, 16, 20; see also Goodman (Ref. 9 ), who has used these equivalence properties in his paper]. Moreover, 
Cesari has proved that, under alternate growth assumptions, the sets $\tilde{Q}(t, x)$ have property $(Q)$. For instance (see Refs. 15,16), if there is a scalar function $\phi(\xi), 0 \leqslant \xi<+\infty$, bounded below, such that $\phi(\xi) / \xi \rightarrow \infty$ as $\xi \rightarrow \infty, z^{0} \geqslant$ $\phi(|x|)$ for all $\left(z^{0}, z\right) \in \tilde{Q}(x)$, and the sets $\tilde{Q}(x)$ are convex and satisfy property $(K)$, then the same sets $\tilde{Q}(x)$ also satisfy property $(Q)$.

We know from the Dunford-Pettis theorem and a remark of De La Valle Poussin that, if $\xi_{k}(t) \rightarrow \xi(t)$ weakly in $L_{1}(G, B)$ as $k \rightarrow \infty$, then necessarily there are a convex function $\phi$ as above and scalar $L$-integrable functions $\sigma(t), \sigma_{k}(t), t \in G$, such that $\sigma_{k}(t) \geqslant \phi\left(\left|\xi_{k}(t)\right|\right)$ and $\sigma_{k}(t) \rightarrow \sigma(t)$ weakly in $L_{1}(G, R)$. If the sets $\tilde{Q}(x)$ above are convex and have property $(K)$, then the sets $\tilde{Q}^{*}(x)=\left[\left(z^{0}, v, u\right): z^{0} \geqslant T(x, u), v \geqslant \phi(u), u \in Q(x)\right]$ are also convex and, by (Refs. 15, 16), have property $(Q)$.

Recently, Suryanarayana (Ref. 21) has shown that any maximal monotone map on a Hilbert space has property $(Q)$.

\section{Duality Operation}

Given an extended real function $T u, u \in R^{n}$ (that is, $T$ may take values $+\infty$ and $-\infty$ ) we denote by epi $T$ (or epigraph of $T$ ) the subset of $R^{n+1}$ defined by

$$
\text { epi } T=\left[\left(z^{0}, u\right) \in R^{n+1} \mid+\infty>z^{0} \geqslant T u\right] .
$$

Thus, the projection of epi $T$ on $R^{n}$ is the set $U$ of all $u$ where $T u<+\infty$. The following well-known statement is needed.

Theorem 5.1. Epi $T$ is closed (in $R^{n+1}$ ) if and only if the extended function $T u$ is lower semicontinuous in $R^{n}$; epi $T$ is convex if and only if $T u$ is convex in $R^{n}$.

If $T u, u \in R^{n}$, is any extended, real-valued function in $R^{n}$, then, for every $v \in R^{n}$, we consider all $r$ real, if any, such that $-r+(v, u) \leqslant T u$, for all $u \in R^{n}$, and we take $T^{*} v=\inf \{r\}$. In other words, we take

$$
T^{*} v=\sup \left[(v, u)-T u \mid u \in R^{n}\right], \quad v \in R^{n} .
$$

Indeed, if $r=T^{*} v$, then $r \geqslant(v, u)-T u$ for all $u$, that is, $T u \geqslant-r+(v, u)$ for all $u$, and $r=T^{*} v$ is the inf of all numbers $r$ for which this holds. Note that, if the statement

$$
-r+(v, u) \leqslant T u, \quad \text { for all } u,
$$

holds for no $r \in R$, that is, the class of such $r$ is empty, then $T^{*} v=-\infty$, according to the usual conventions. The following further statement is needed. Here, $T^{*}$ is said to be the dual of $T$. 
Theorem 5.2. If $T u, u \in R^{n}$, is an extended real-valued function, $T u \neq \equiv+\infty, T u \neq-\infty$ for all $u$, and $T u$ is convex and lower semicontinuous in $R^{n}$, then

$$
\text { epi } T=\bigcap_{v \in R^{n}}\left[\left(z^{0}, u\right) \mid z^{0} \geqslant(v, u)-T^{*} y\right] \text {. }
$$

Moreover, $T^{*} v, v \in R^{n}$, is also an extended real-valued function, $T^{*} v \neq+\infty$, $T^{*} v \neq-\infty$ for all $v$, and $T^{*} v$ is convex and lower semicontinuous in $R^{n}$. Finally, $\left(T^{*}\right)^{*}=T$, or briefly $T^{* *}=T$.

For functions $T(x, u)$ depending on $u$ and also on the parameter $x$, with the usual conventions, we shall understand that all duality operations are made with respect to the second variable. On the other hand, as before, we shall denote by $\tilde{Q}(x)$ the set of all $\left(z^{0}, u\right) \in R^{n+1}$ with $+\infty>z^{0} \geqslant T(x, u)$.

For integrand functions $F(t, x, u)$ of problems of optimization, the dual with respect to $u$, namely $F^{*}(t, x, p)$, is of course the Hamiltonian.

Given any function $T$ as above, we denote by $\mathrm{cl} T$ the new analogous function such that

$$
\text { epi }(\mathrm{cl} T)=\mathrm{cl}(\operatorname{epi} T) \text {. }
$$

Property $(Q)$ of the sets $Q(x)$ can now be equivalently expressed in terms of duality operations as Goodman has recently proved (Ref. 9),

Theorem 5.3. (Goodman). If $T(x, u) \neq-\infty$ for all $x, u$, then the sets $\tilde{Q}(x)$ have property $(Q)$ at $x_{0}$ if and only if

$$
T^{*}\left(x_{0}, v\right)=\mathrm{cl}\left[\lim _{x \rightarrow x_{0}} \sup T^{*}(x, v)\right] .
$$

Theorem 5.4. (Goodman). If $T(x, u) \neq-\infty$ for all $x$ and $u$, then the sets $\dot{Q}(x)$ have property $(Q)$ at $x_{0}$ if and only if

$$
T\left(x_{0}, y\right)=\left[\lim _{x \rightarrow x_{0}} \sup T^{*}(x, y)\right]^{*} .
$$

For further necessary and sufficient conditions for property $(Q)$ in terms of duality and lattice operations, we refer to Ref. 9 and to the exposition in Ref. 15.

For integrand functions $F_{0}(t, x, z)$ which are convex in $(x, z)$, then, for every $t$, the dual operation above in the variable $(x, z)$ yields a dual function $F_{0}^{*}(t, p, w)$ with $F_{0}^{* *}=F_{0}$. The corresponding dual problems (say, $I$ and $I^{*}$ ) possess formal symmetric dual properties in the class of functions, say, $y(t)=y_{1}(t)+y_{2}(t), y_{1}$ absolutely continuous as usual, while $y_{2}$ is of bounded variation and singular. Lower-closure theorems hold with suitable topologies as mentioned in Section 3, and the dual properties of $I$ and $I^{*}$ are then proved as usual. 


\section{Analytical Property (D)}

We shall use here the actual notations of optimal control theory, where explicit use is made of control parameters and control functions or strategies. Let $A$ be a given closed subset of the $t y$-space $R^{n+1}$, whose projection on the $t$-axis is an interval $I$ finite or infinite. For every $(t, y) \in A$, let $U(t, y)$ be a given subset of the $u$-space $R^{m}$, and let $M$ denote the set of all $(t, y, u)$ with $(t, y) \in A, u \in U(t, y)$. We denote by $A(t)$ the sections of $A$, that is, the sets

$$
A(t)=[x \mid(t, y) \in A] \subset R^{n}, \quad t \in I .
$$

Let

$$
f_{0}(t, y, u), f(t, y, u)=\left(f_{1}, \ldots, f_{n}\right)
$$

be given functions defined on $M$. Here, $u$ denotes the control variable and $U(t, y)$ the control set function. Let $\tilde{f}$ denote the function

$$
\tilde{f}(t, y, u)=\left(f_{0}, f_{1}, \ldots, f_{n}\right) \text {. }
$$

For any $(t, y) \in A$, let $Q(t, y)$ and $\tilde{Q}(t, y)$ denote the sets

$$
\begin{aligned}
& Q(t, y)=f(t, y, U(t, y))=[z \mid z=f(t, y, u), u \in U(t, y)] \subset R^{n}, \\
& \tilde{Q}(t, y)=\left[\left(z^{0}, z\right) \mid z^{0} \geqslant f_{0}(t, y, u), z=f(t, y, u), u \in U(t, y)\right] \subset R^{n+1},
\end{aligned}
$$

so that $Q(t, y)$ is the projection of $\tilde{Q}(t, y)$ on the $z$-space $R^{n}$. Note that, if

$$
T(t, y, z)=\inf \left[z^{0} \mid\left(z^{0}, z\right) \in \tilde{Q}(t, y)\right], \quad(t, y, z) \in M,
$$

then $T(t, y, z)<+\infty$ in $M$. We may extend $T$ to all of $R^{1+2 n}$ by taking $T(t, y, z)=+\infty$ in $R^{1+2 n}-M$. Thus, $T$ is one of the functions that we considered above. Note that, if all the sets $\tilde{Q}(t, y)$ are closed, then whenever $T(t, y, z)$ is finite, then min can replace inf in Eq. (4) or, equivalently,

$$
\tilde{Q}(t, y)=\left[\left(z^{0}, z\right) \mid z^{0} \geqslant T(t, y, z), z \in Q(t, y)\right] .
$$

A number of criteria for property $(Q)$ of the sets $\tilde{Q}(t, y)$ have been proved by Cesari and others (Refs. $7,8,15,16$ ). Below, we present a set of analytical considerations in finite-dimensional spaces which imply property $(Q)$, and which have natural extensions in Banach spaces. We shall assume here that the set $U(t)$ depend on $t$ only.

Let $u_{k}(t), u(t), y_{k}(t), y(t), t \in G=[a, b], k=1,2, \ldots$, be measurable functions, $u_{k}(t) \in U(t), y_{k}(t) \in A(t)$, and let $\tilde{\delta}_{k}=\left(\delta_{k}^{0}, \delta_{k}\right)$ be defined by

$$
\tilde{\delta}_{k}(t)=\tilde{f}\left(t, y_{k}(t), u_{k}(t)\right)-\tilde{f}\left(t, y(t), u_{k}(t)\right), \quad t \in[a, b] .
$$

Proposition 6.1. $\tilde{\delta}_{k}(t) \rightarrow 0$ strongly in $\left(L_{1}(G)\right)^{n+1}$ if and only if the same functions $\tilde{\delta}_{k}(t)$ are equiabsolutely integrable in $G$. 
This statement was proved in Ref. 10 in a slightly more elementary situation, but the proof is the same.

Proposition 6.2. $\quad \tilde{\delta}_{k}(t) \rightarrow 0$ strongly in $\left(L_{1}(G)\right)^{n+1}$ implies the following weak form of property $(Q)$; namely, there is a subsequence $\left[k_{s}\right]$ such that

$$
\tilde{Q}(t, y(t)) \supset \bigcap_{h=1}^{\infty} \operatorname{cl} \operatorname{co}\left[\bigcup_{s=h}^{\infty} \tilde{f}\left(t, y_{k_{s}}(t), u_{k_{s}}(t)\right)\right], \quad t \in G \text { a.e. }
$$

This statement was proved in Refs. 10 and 3, Part 1 , in various settings. The proof in the present situation is the same. In Ref. 1, p. 352 (cf. also Ref. $10)$, we have pointed out that, for lower closure theorems, it is enough to assume only that $\delta_{k} \rightarrow 0$ strongly in $\left(L_{1}(G)\right)^{n}$ and that

$$
\infty>\lim \inf _{k \rightarrow \infty} \int_{G} \delta_{k}^{0}(t) d t \geqslant 0 .
$$

For mere closure theorems, $f_{0}=0$, and the last requirement is trivial.

We denote by property $(D)$ of a sequence $y_{k}(t), u_{k}(t), t \in G, k=$ $1,2, \ldots$, as above the requirement $\tilde{\delta}_{k}(t) \rightarrow 0$ strongly in $\left(L_{1}(G)\right)^{n+1}$, or $\left\|\tilde{\delta}_{k}\right\| \rightarrow 0$.

Below we give several specific criteria for property $(D)$. This property implies the weak form of property $(Q)$ stated above. We have mentioned these criteria in Refs. 2, 10, 22, and Ref. 3, Part I. They are repeated here in the present context, since they hold not only in Euclidean spaces but also in Banach space situations. [see Ref. 10, pp. $446-447$, properties $\left(F_{p}\right)$ and $\left.\left(F_{\infty}\right)\right]$.

(Lg) Lipschitz type conditions, geometric viewpoint.

$\left(F_{p}\right), 1 \leqslant p<\infty$. Here, $B$ and $Y$ are Banach spaces, $U$ a metric space. If $1 \leqslant p<\infty$, let us assume that there are constants $c, \gamma, \xi_{0}, p^{\prime}, c \geqslant 0, \xi_{0} \geqslant 0$, $0<\gamma \leqslant p, p^{\prime}=p(p-\gamma)^{-1}$ if $0<\gamma<p, p^{\prime}=\infty$ if $\gamma=p$, and functions $F(t) \geqslant$ $0, t \in G, F \in L_{p^{\prime}}(G, R), h(\xi), 0 \leqslant \xi<\infty, h$ monotone nondecreasing, $h(0+)=0, h(\xi) \leqslant c|\xi|^{\gamma}$ for $\xi \geqslant \xi_{0}$, such that, for all $\left(t, y_{1}, u\right),\left(t, y_{2}, u\right) \in M$, $t \in G-T_{0}$, meas $T_{0}=0$, we have

$$
\left\|\tilde{f}\left(t, y_{1}, u\right)-\tilde{f}\left(t, y_{2}, u\right)\right\|_{B} \leqslant F(t) h\left(\left\|y_{1}-y_{2}\right\|_{Y}\right) .
$$

Let $\xi(t), y(t), \eta_{k}(t), \bar{\eta}_{k}(t), \xi_{k}(t), \quad \bar{\xi}_{k}(t), \quad y_{k}(t), \quad u_{k}(t), t \in G, k=$ $1,2, \ldots$, be such that

$$
\begin{gathered}
y(t), y_{k}(t) \in A(t), \quad u(t), u_{k}(t) \in U(t), \\
\eta_{k}(t) \geqslant f_{0}\left(t, y_{k}(t), u_{k}(t)\right), \quad \xi_{k}(t)=f\left(t, y_{k}(t), u_{k}(t)\right), \\
\bar{\eta}_{k}(t) \geqslant f_{0}\left(t, y(t), u_{k}(t)\right), \quad \bar{\xi}_{k}(t)=f\left(t, y(t), u_{k}(t)\right), \quad t \in G, \quad k=1,2, \ldots, \\
\xi_{k} \rightarrow \xi \text { weakly in } L_{1} .
\end{gathered}
$$


Let us assume that, for almost all $t \in G$, the sets $Q(t, y(t))$ are closed and convex and that $\left\|y_{k}-y\right\|_{p} \rightarrow 0$ as $k \rightarrow \infty$. Then, property $(Q)$ holds as in Proposition 6.2 for almost all $t \in G$, and the lower-closure property holds as in Theorem 3.1.

Analogously, we have property $\left(F_{\infty}\right)$ :

$\left(F_{\infty}\right)$ Let $B, U, Y$ be as above. Let us assume that there are functions $F(t) \geqslant 0, t \in G, F \in L_{1}(G, R)$, such that (5) holds. Let $\xi(t), y(t), \eta_{k}(t), \bar{\eta}_{k}(t)$, $\xi_{k}(t), \bar{\xi}_{k}(t), y_{k}(t), u_{k}(t), t \in G, k=1,2, \ldots$, be as in Remark 3.4; assume that, for a.a. $t \in G$, the set $\tilde{Q}(t, y(t))$ is closed and convex and that $\left\|y_{k}-y\right\|_{\infty} \rightarrow$ 0 as $k \rightarrow \infty$. Then, property $(D)$ holds, and then also property $(Q)$ holds as in Proposition 6.2 for almost all $t \in G$, and the lower-closure theorem holds as in Theorem 3.1.

(Lf) Lipschitz type conditions, functional viewpoint.

$\left(F_{p}^{\prime}\right), 1 \leqslant p<\infty$. Let $\{u(t)\}$ be a family of control functions, and let us assume that, for every control function $u=u(t)$ of the class, there is a function $F_{u}(t)$ with the following properties. First, let $p, c, \gamma, \xi_{0}, p^{\prime}, h$ as in $\left(F_{p}\right)$, and let $F_{u}(t) \geqslant 0, t \in G, F_{u} \in L_{p^{\prime}}(G, R),\left\|F_{u}\right\|_{p^{\prime}} \leqslant M$, and

$$
\left\|\tilde{f}\left(t, y_{1}, u(t)\right)-\tilde{f}\left(t, y_{2}, u(t)\right)\right\|_{B} \leqslant F_{u}(t) h\left(\left\|y_{1}-y_{2}\right\|_{Y}\right) .
$$

Let $\xi(t), y(t), \eta_{k}(t), \bar{\eta}_{k}(t), \xi_{k}(t), \bar{\xi}_{k}(t), y_{k}(t), u_{k}(t), t \in G, k=1,2, \ldots$, be as in (Lg) with $u_{k} \in\{u(t)\}$ for all $k$; assume that, for all $t \in G$, the sets $Q(t, y(t))$ are closed and convex and that $\left\|y_{k}-y\right\|_{p} \rightarrow 0$ as $k \rightarrow \infty$. Then, property $(Q)$ holds as in Proposition 6.2, and the lower-closure theorem holds as in Theorem 3.1.

In particular $F$ may be simply a function of $(t, u)$, say $F=F(t, u)$, or $F_{u}(t)=F(t, u(t))$, and in this case we need require that

$$
\int_{G}\|F(t, u(t))\|^{p^{\prime}} d t \leqslant M
$$

Analogously, we have property $\left(F_{\infty}^{\prime}\right)$ :

$\left(F_{\infty}^{\prime}\right)$ Let $B, Y, U$ be as above. Let us assume that there is a function $h(\xi), 0 \leqslant \xi<+\infty, h$ monotone nondecreasing, $h(0+)=0$, and, for every $u \in\{u(t)\}$, a function $F_{u}(t) \geqslant 0, F_{u} \in L_{1}(G, R),\left\|F_{u}\right\|_{1} \leqslant M$, such that (6) holds for all $u \in\{u(t)\}$. Let $\xi(t), y(t), \eta_{k}(t), \bar{\eta}_{k}(t), \xi_{k}(t), \bar{\xi}_{k}(t), y_{k}(t), u_{k}(t), t \in G$, $k=1,2, \ldots$, be as in property $\left(F_{p}^{\prime}\right)$, with $u_{k} \in\{u(t)\}$ for all $k$, and assume that, for a.a. $t \in G$, the set $Q(t, y(t))$ is closed and convex and that $\left\|y_{k}-y\right\|_{\infty} \rightarrow$ 0 as $k \rightarrow \infty$. Then, property $(Q)$ holds as in Proposition 6.2, and the lower-closure theorem holds as in Theorem 3.1.

In particular, $F$ may be simply a function of $(t, u)$, say $F=F(t, u)$, or $F_{u}(t)=F(t, u(t))$, and in this case we need require that

$$
\int_{G} F(t, u(t)) d t \leqslant M
$$


For a great many other analytical criteria for property $(Q)$, we refer to Ref. 2, Ref. 3, Part 1, and Refs. 8, 15, 23.

Remark 6.1. Berkovitz (Ref. 24) and Bates (Ref. 25) state that, in their lower-closure theorems, property $(Q)$ does not hold. They assume a Lipschitz condition

$$
\left|\tilde{f}\left(t, y_{1}, u\right)-\tilde{f}\left(t, y_{2}, u\right)\right| \leqslant F(t, u) h\left(\left|y_{1}-y_{2}\right|\right)
$$

as above and the further assumption

$$
\int_{t_{1}}^{t_{2}} F(t, u(t)) d t \leqslant A
$$

From the above, it is apparent that property $(Q)$ in the weak form does hold in their situation. Both Berkovitz and Bates do not seem to have realized this in their statements. Their proofs are not much different from ours in the analogous context.

\section{Appendix}

In connection with Theorem 2.1 , let us prove here directly that $(\mathrm{G} 2)$ implies weak compactness in $H^{1,1}$. First, for $\epsilon=1$ we have $\left|x^{t}(t)\right| \leqslant$ $\Psi_{1}(t)+\eta(t) ;$ hence, $\eta(t) \geqslant-\Psi_{1}(t)$. Let

$$
M_{0}=\int_{a_{0}}^{b_{0}} \Psi_{1}(t) d t
$$

Now, given $\epsilon>0$, let $\sigma=\min \left[1, \epsilon 2^{-1}\left(M_{0}+M_{1}+1\right)^{-1}\right]$, and note that the function $\Psi_{0}(t)$ is $L$-integrable in $\left[a_{0}, b_{0}\right]$. Hence, there is some $\delta>0$, such that

$$
\int_{E} \Psi_{\sigma}(t) d t<\epsilon / 2
$$

for every measurable subset $E$ of $\left[a_{0}, b_{0}\right]$ with meas $E<\delta$. Finally, let $\eta(t)$, $x(t), a \leqslant t \leqslant b$, be any pair of the family, and let $E$ be any measurable subset of $[a, b]$ with meas $E<\delta$. Then

$$
\begin{aligned}
\int_{E}\left|x^{\prime}(t)\right| d t & \leqslant \int_{E}\left[\Psi_{\sigma}(t)+\sigma \eta(t)\right] d t \leqslant \int_{E}\left[\Psi_{\sigma}+\sigma\left(\eta+\Psi_{1}\right)\right] d t \\
& \leqslant \sigma \int_{b}^{a} \eta d t+\sigma \int_{a}^{b} \Psi_{1} d t+\int_{E} \Psi_{\sigma} d t \\
& \leqslant \sigma\left(M_{0}+M_{1}\right)+\int_{E} \Psi_{\sigma} d t \leqslant \epsilon / 2+\epsilon / 2=\epsilon .
\end{aligned}
$$


This proves the absolute integrability of the class $\left\{x^{\prime}\right\}$. The proof continues as in Section 2.

\section{References}

1. CESARI, L., Closure Theorems for Orientor Fields and Weak Convergence, Archive for Rational Mechanics and Analysis, Vol. 55, pp. 332-356, 1974.

2. CeSARI, L., and SURYANARAYANA, M. B., Nemitsky's Operators and Lower Closure Theorems, Journal of Optimization Theory and Applications, Vol. 19, pp. 165-183, 1976.

3. CESARI, L., Geometric and Analytic Views in Existence Theorems for Optimal Control in Banach spaces, $I, I I$, and III, Journal of Optimization Theory and Applications, Vol. 14, pp. 505-520, 1974; Vol. 15, pp. 467-497, 1975; and Vol. 19, pp, 185-214, 1976.

4. Cesari, L., and SuryanarayanA, M. B., An Existence Theorem for Pareto Problems, Nonlinear Analysis, Theory, Methods, and Applications, Vol. 2, pp. 225-233, 1978.

5. Cesari, L., and Suryanarayana, M. B., Existence Theorems for Pareto Optimization. Multivalued and Banach Space Valued Functionals, Transactions of the American Mathematical Society, Vol. 244, pp. 37-65, 1978.

6. KAISER, P., and SuryanARAyAnA, M. B., Orientor Field Equations in Banach Spaces, Journal of Optimization Theory and Applications, Vol. 19, pp. 141-164, 1976.

7. CESARI, L., Seminormality and Upper Semicontinuity in Optimal Control, Journal of Optimization Theory and Applications, Vol. 6, pp. 114-137, 1970.

8. CESARI, L., Closure, Lower Closure and Semicontinuity Theorems in Optimal Control, SIAM Journal on Control, Vol. 9, pp. 287-315, 1971.

9. GoOdMAN, G. S., The Duality of Convex Functions and Cesari's Property $(Q)$, Journal of Optimization Theory and Applications, Vol. 19, pp. 17-23, 1976.

10. CESARI, L., and SURYANARAYANA. M. B., Closure Theorems without Seminormality Conditions, Journal of Optimization Theory and Applications, Vol. 15, pp. 441-465, 1975.

11. MCShANE, E. J., Integration, Princeton University Press, Princeton, New Jersey, 1944.

12. Cesari, L., LA Palm, J. R., and Nishiura, T., Remarks on Some Existence Theorems for Optimal Control, Journal of Optimization Theory and Applications, Vol. 3, pp. 296-305, 1969.

13. CECCONI, J., Sul Calcolo delle Variazioni, Bolletino della Unione Matematica Italiana, Serie 5, Vol. 15 A, pp. 279-299, 1978.

14. ROCKAFELLAR, R. T., Integral Functionals, Normal Integrands, and Measurable Selections, Nonlinear Operators and the Calculus of Variations, Edited by J.

P. Gossez et al, Springer-Verlag, Berlin, Germany, pp. 157-207, 1976.

15. CesARI, L., Problems of Optimization, Springer-Verlag, Berlin, Germany, 1981. 
16. CESARI, L., Existence Theorems of Optimal Control of Mayer Type, SIAM Journal on Control, Vol, 6, pp. 517-552, 1968.

17. SURYANARAYANA, M. B., Remarks on Lower Semicontinuity and Lower Closure, Journal of Optimization Theory and Applications, Vol. 19, pp. 125140, 1976.

18. CESARI, L., Lower Semicontinuity and Lower Closure Theorems without Seminormality Conditions, Annali di Matematica Pura e Applicata, Vol. 98, pp. 381-397, 1974.

19. OLECH, C., Review No. 1169, Mathematical Reviews, Vol. 56, p. 168, 1978.

20. CESARI, L., Existence Theorems for Weak and Usual Solutions in Lagrange Problems with Unilateral Constraints, I and II, Transactions of the American Mathematical Society, Vol. 124, pp. 369-412 and pp. 413-430, 1966.

21. SURYANARAYANA, M. B., Monotonicity and Upper Semicontinuity, Bulletin of the American Mathematical Society, Vol. 82, pp. 936-938, 1976.

22. CeSARI, L., and SURYANARAYANA, M. B., Convexity and Property $(Q)$ in Optimal Control Theory, SIAM Journal on Control, Vol. 12, pp.705-720, 1974.

23. CESARI, L., and SURYANARA YANA, M. B., Upper Semicontinuity Properties of Set-Valued Functions, Nonlinear Analysis, Theory, Methods, and Applications, Vol. 4, pp. 639-656, 1980.

24. Berkovitz, L., Optimal Control Theory, Springer-Verlag, Berlin, Germany, 1974.

25. BATES, G. R., Lower Closure and Existence Theorems for Optimal Control Problems with Infinite Horizon, Journal of Optimization Theory and Applications, Vol. 24, pp. 639-649, 1978.

26. IOFFE, A. D., On Lower Semicontimuty of Integral Functionals, I and II, SIAM Journal on Control, Vol. 15, pp. 521-538 and pp. 991-1000, 1977.

27. OLeCH C., Existence Theorems for Optimal Control with Vector-Valued Cost Functions, Transactions of the American Mathematical Society, Vol. 136, pp. 157-180, 1969.

28. OLECH, C., Existence Theory in Optimal Control Problems: The Underlying Ideas, Proceedings of the International Conference on Differential Equations, Edited by H. A. Antosiewicz, Academic Press, New York, New York, 1975.

29. OLECH, C., Weak Lower Semicontinuity of Integral Functionals, Journal of Optimization Theory and Applications, Vol. 19, pp. 3-16, 1976.

30. LAsotA, A., and OLECH, C., On Cesari's Semicontinuity Condition for SelValued Mappings, Bulletin de l'Academie Polonaise de Sciences, Série des Sciences Mathématiques, Astronomiques, et Physiques, Vol. 16, pp. 705-720, 1974. 